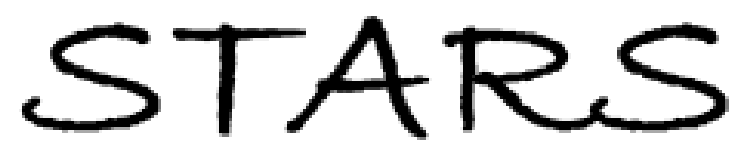

University of Central Florida

STARS

Faculty Bibliography 2000s

Faculty Bibliography

$1-1-2006$

\title{
Solitons in dispersion-inverted AlGaAs nanowires
}

\author{
R. El-Ganainy \\ University of Central Florida \\ S. Mokhov \\ University of Central Florida \\ K. G. Makris \\ University of Central Florida \\ D. N. Christodoulides \\ University of Central Florida \\ R. Morandotti
}

Find similar works at: https://stars.library.ucf.edu/facultybib2000

University of Central Florida Libraries http://library.ucf.edu

This Article is brought to you for free and open access by the Faculty Bibliography at STARS. It has been accepted for inclusion in Faculty Bibliography 2000s by an authorized administrator of STARS. For more information, please contact STARS@ucf.edu.

\section{Recommended Citation}

El-Ganainy, R.; Mokhov, S.; Makris, K. G.; Christodoulides, D. N.; and Morandotti, R., "Solitons in dispersioninverted AIGaAs nanowires" (2006). Faculty Bibliography 2000 s. 6114.

https://stars.library.ucf.edu/facultybib2000/6114

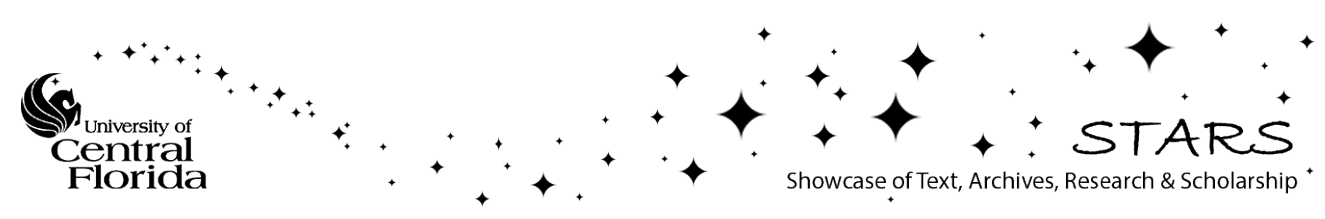




\title{
Solitons in dispersion-inverted $A l G a A s$ nanowires
}

\author{
R. El-Ganainy, S. Mokhov, K. G. Makris, and D. N. Christodoulides \\ College of Optics \& Photonics-CREOL \& FPCE, University of Central Florida, Orlando, Florida, 32816
}

\section{R. Morandotti}

Institut national de la recherché scientifique, Université du Québec, Varennes, Québec, Canada J3X IS2

\begin{abstract}
We demonstrate that optical solitons can exist in dispersioninverted highly-nonlinear AlGaAs nanowires. This is accomplished by strongly reversing the dispersion of these nano-structures to anomalous over a broad frequency range. These self-localized waves are possible at very low power levels and can form in millimeter long nanowire structures. The intensity and spectral evolution of solitons propagating in such AlGaAs nanowaveguides is investigated in the presence of loss, multiphoton absorption and higher-order dispersion.

(C)2006 Optical Society of America

OCIS codes: (190.5530) pulse propagation and solitons, (190.4370) Nonlinear optics, fibers, (260.2030) Dispersion.
\end{abstract}

\section{References and links}

1. P. N. Prasad, Nanophotonics, (John Wiley and Sons, New York 2004).

2. S. G. Leon-Saval, T. A. Birks, W. J. Wadsworth, P. St. J. Russell, and M. W. Mason, "Supercontinuum generation in submicron fibre waveguides," Opt. Express 12, 2864-2869 (2004).

3. L. Tong, R. R. Gattass, J. B. Ashcom, S. He, J. Lou, M. Shen, I. Maxwell, and E. Mazur, "Subwavelengthdiameter silica wires for low-loss optical wave guiding", Nature 426, 816-819, (2003).

4. J.C.Knight, J. Arriaga, T.A Briks, A. Ortigosa-Blanch, W.J. Wadsworth and P. St. J. Russell, “ Anomalous Dispersion in Photonic Crystal Fiber”, IEEE Photonics Technology Letters 12, 807-809, (2000).

5. M. A. Foster, A. L. Gaeta, Q. Cao, and R. Trebino, "Soliton-effect compression of supercontinuum to few-cycle durations in photonic nanowires," Opt. Express 13, 6848-6855 (2005).

6. G. Brambilla, F. Koizumi, V. Finazzi, and D. J. Richardson, "Supercontinuum generation in tapered bismuth silicate fibres", Electron. Lett. 41, 795-797 (2005).

7. H. Ebendorff-Heidepriem, P. Petropoulos, S. Asimakis, V. Finazzi, R. C. Moore, K. Frampton, F. Koizumi, D. J. Richardson, and T. M. Monro, "Bismuth glass holey fibers with high nonlinearity", Opt. Express 12, 50825087 (2004).

8. G. Brambilla, F. Koizumi, X. Feng, and D. J. Richardson, "Compound-glass optical nanowires", Electron. Lett. 41, 400-402 (2005).

9. H. Fukuda, K. Yamada, T. Shoji, M. Takahashi, T. Tsuchizawa, T. Watanabe, J. Takahashi and S. Itabashi, "Four-wave mixing in silicon wire waveguide", Opt. Express, 13, 4629-4635, (2005).

10. Y. K. Lizé, E. C. Mägi, V. G. Ta'eed, J. A. Bolger, P. Steinvurzel, and B. J. Eggleton, "Microstructured optical fiber photonic wires with subwavelength core diameter", Opt. Express 12, 3209-3217 (2004).

11. V. Kumar, A. George, J. C. Knight, and P. St. J. Russell, "Tellurite photonic crystal fiber", Opt. Express 11, 2641-2645 (2003).

12. J. C. Knight, "Photonic Crystal fibers", Nature 424, 847-851 (2003).

13. G.I. Stegeman, A. Villeneuve, J. Kang, J.S. Aitchison, C.N. Ironside, K. Al-hemyari, C.C. Yang, C-H. Lin, HH. Lin, G.T. Kennedy, R.S. Grant and W. Sibbett, "AlGaAs Below Half Bandgap: The Silicon of Nonlinear Optical Materials", Int. J. of Nonlinear Optical Physics, 3, 347-371 (1994).

14. J. E. Heebner, N. N. Lepeshkin, A. Schweinsberg, G. W. Wicks, R. W. Boyd, R. Grover and P.-T. Ho, "Enhanced linear and nonlinear optical phase response of AlGaAs microring resonators", Opt. Lett. 29, 769 $771,(2004)$.

15. R. Iwanow, G. I. Stegeman, D. N. Christodoulides, R. Morandotti, D. Modotto, A. Locatelli, C. De Angelis, F. Pozzi, C. R. Stanley, and M. Sorel, "Enhanced Third-Order Nonlinear Effects in Optical AlGaAs Nanowires", post-deadline paper PDP7, Nonlinear Guided Waves and Their Applications, OSA Topical Meeting, Dresden, Germany, Sep. 6-9, 2005.

\#67969 - \$15.00 USD

(C) 2006 OSA
Received 10 February 2006; revised 10 March 2006; accepted 11 March 2006 20 March 2006 / Vol. 14, No. 6 / OPTICS EXPRESS 2277 
16. U. Peschel, T. Peschel, and F. Lederer, "A compact device for highly efficient dispersion compensation in fiber transmission”, Appl. Phys. Lett. 67, 2111-2113 (1995).

17. C.J. Hamilton, B. Vogele, J.S. Aitchison, G.T. Kennedy, W. Sibbett, W. Biehlig, U. Peschel, T. Peschel, F. Lederer, "Bright Solitary pulses in AlGaAs waveguides at half the band gap", Opt. Lett. 21, 1226-1228, (1996).

18. S.Gehrsitz, F.K. Reinhart, C. Gourgon, A. Vonlanthen and H. Sigg, "The refractive index of $\mathrm{Al}_{\mathrm{x}} \mathrm{Ga}_{1-\mathrm{x}} \mathrm{As}$ below the band gap: Accurate determination and empirical modeling” J. Appl. Phys, 87,7825-7837 (2000).

19. J. U. Kang, A. Villeneuve, M. Sheik-Bahae, George I. Stegeman, K. Al-hemyari, J. S. Aitchison, C. N. Ironside, "Limitation due to three-photon absorption on the useful spectral range for nonlinear optics in AlGaAs below half band gap", Appl. Phys. Lett. 65, 147-149 (1994).

20. K. Okamoto, Fundamental of Optical Waveguides, (Academic Press, San Diego 2000).

21. L. Tong, J. Lou, and E. Mazur, "Single-mode guiding properties of subwavelength-diameter silica and silicon waveguides", Opt. Express 12,1025-1035 (2004).

22. A.L. Gaeta, "Nonlinear propagation and continuum generation in microstructured optical fibers"Opt. Lett. 27, 924-926 (2002).

\section{Introduction}

The interaction of light with matter on a nanometer scale opens up new opportunities which may have far reaching implications in telecommunications, computation, biophotonics, and sensing technologies [1]. Lately, single-mode sub-wavelength waveguides or nanowires have been realized in several material systems [2-11]. Such optical nanowire structures are capable of providing superior light confinement and are thus ideal for nonlinear optics applications [12]. Clearly, of interest will be to fabricate such nanowires using high contrast, highly nonlinear materials, such as $A l G a A s$-known to exhibit a nonlinearity that is three orders of magnitude higher than that of silica glass [13,14]. Apart from being highly nonlinear, AlGaAs nanowires are also highly promising in terms of applications since they can be integrated with other optoelectronic components on the same wafer and thus can serve as information conduits among miniaturized devices. Quite recently, enhanced spectral broadening or SPM has been observed for the first time in 700 $\mu \mathrm{m}$ long AlGaAs nanowires [15]. An important question associated with this particular system is whether optical solitons are possible in AlGaAs nanowaveguides. This is of relevance since solitons can be used to either overcome dispersion effects or to achieve pulse compression in such nanostructures. We note that so far, optical solitons have only been observed in multi-layer AlGaAs structures in which the dispersion can be engineered [16,17]. Yet, in primitive weakly guiding $A l G a A s$ waveguides such solutions are not possible since this material system exhibits appreciable normal dispersion in the spectral region of interest [18], i.e. for $1.1 \mu m<\lambda<1.7 \mu m$. Here we theoretically demonstrate, that, because of high contrast, the dispersion of an air-clad AlGaAs nanowire can become strongly anomalous (becomes inverted), thus overcoming material dispersion limitations. This in turn may allow optical soliton formation in millimeter long structures. These solitons are possible at very low power levels (at $\sim 5 \mathrm{~W}$ ) in spite of the fact that the AlGaAs nanowires can exhibit anomalous dispersion that is a thousand times higher than that of silica glass. The intensity and spectral evolution of these solitons is investigated in AlGaAs nanowaveguides in the presence of loss, multi-photon absorption, and higher-order dispersive and nonlinear effects.

\section{AlGaAs Nanowire dispersion properties}

To analyze the dispersion properties of $A l G a A s$ nanowires, let us consider for example an $A l_{0.2} G a_{0.8} A s$ nanorod of core radius $a$ as shown in Fig.1(a). This particular composition ( $\left.A l_{0.2} G a_{0.8} A s\right)$ is deliberately chosen since in the neighborhood of $\lambda=1.55 \mu m$ it is known to exhibit relatively low two-photon absorption [19]. In addition, we assume that the nanowire is air-cladded. We note that even though completely air-clad structures are rather difficult to develop these days, it is yet possible to fabricate waveguides that are mostly surrounded by air, with characteristics very similar to the one analyzed here. At this wavelength the

\#67969 - \$15.00 USD

(C) 2006 OSA
Received 10 February 2006; revised 10 March 2006; accepted 11 March 2006 20 March 2006 / Vol. 14, No. 6 / OPTICS EXPRESS 2278 
refractive index of $A l_{0.2} G a_{0.8} A s$ is approximately 3.27, and the nanorod is operated at or close to the single-mode regime. In general, the nanowire dispersion relation is given by [20]:

$$
\left[\frac{J_{m}^{\prime}(u)}{u J_{m}(u)}+\frac{K_{m}^{\prime}(w)}{w K_{m}(w)}\right]\left[\frac{J_{m}^{\prime}(u)}{u J_{m}(u)}+\left(\frac{n_{\text {clad }}}{n_{\text {core }}}\right)^{2} \frac{K_{m}^{\prime}(w)}{w K_{m}(w)}\right]=m^{2}\left(\frac{1}{u^{2}}+\frac{1}{w^{2}}\right)\left(\frac{1}{u^{2}}+\left(\frac{n_{\text {clad }}}{n_{\text {core }}}\right)^{2} \frac{1}{w^{2}}\right)
$$

In Eq.(1) $J_{m}(u)$ is a Bessel function of the first kind and of order $m$ and similarly $K_{m}(w)$ is a modified Bessel function, and primes represent derivatives with respect to the argument. $u=a k_{o} \sqrt{n_{\text {core }}^{2}-n_{\text {eff }}^{2}}, w=a k_{o} \sqrt{n_{\text {eff }}^{2}-n_{\text {clad }}^{2}}$ where $k_{0}=2 \pi / \lambda$ is the free space wavevector and $n_{\text {eff }}$ is the effective refractive index of the mode [20]. $n_{\text {core }}$ is the core index and $n_{\text {clad }}=1$ since the exterior medium is air. The wavelength dependence of the refractive index is accounted by using the Sellmeier expansion of $A l_{x} G a_{1-x} A s$, i.e [18]:

$$
n_{\text {core }}^{2}(x, T, \lambda)=A(x, T)+\frac{C_{o}(x)}{E_{o}^{2}(x, T)-1 / \lambda^{2}}+\frac{C_{1}(x)}{E_{1}^{2}(x, T)-1 / \lambda^{2}}
$$

In general, the constants $A, C_{0}, C_{1}, E_{0}$ and $E_{1}$ depend on the $A l$ composition $(x)$, the temperature $T$, and the free space wavelength $\lambda$. Here all calculations were carried out at room temperature.

The dispersion properties of the fundamental mode $H E_{11}$ of such a nano-structure are obtained by numerically solving Eq. (1) for $m=1$, with the core index evaluated at each frequency step from Eq. (2). The dispersion coefficient $\beta^{\prime \prime}=d^{2} \beta / d \omega^{2}$ of an $A l_{0.2} G a_{0.8} A s$ nanowire is shown in Fig.1(b) in units of $\mathrm{ps}^{2} / \mathrm{m}$ for different core radii. In this same figure, the bulk $A l_{0.2} G a_{0.8} A s$ dispersion is also included for comparison.
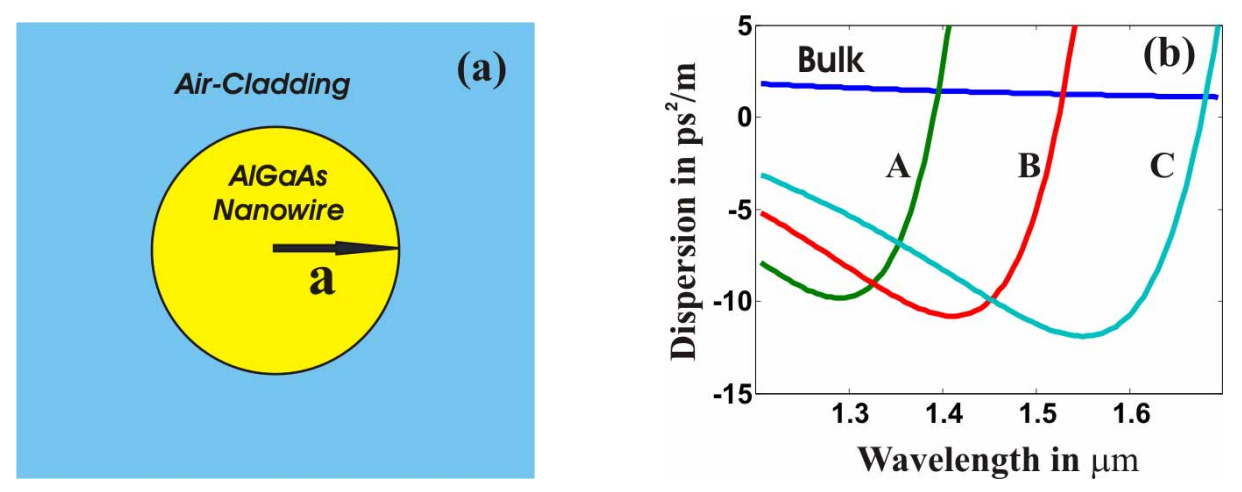

Fig. 1. (a) a nanowire structure; (b) group velocity dispersion $\beta^{\prime \prime}$ of an $A l_{0.2} G a_{0.8} A s$ nanowire when its radius is (A) 160, (B) 175 and (C) $193 \mathrm{~nm}$. Bulk dispersion of $A l_{0.2} G a_{0.8} A s$ is also shown.

It is important to note that because of the strong index contrast of the $A l G a A s$ nanowaveguide and the resulting field confinement, the waveguide dispersion dominates and as a result the dispersion can become inverted and strongly anomalous [4,21]. In fact for $a=193 \mathrm{~nm}$, the dispersion of such a nanorod can reach very high values, as high as $-12 p s^{2} / m$, which is $10^{3}$ times higher than that of a standard silica fiber at $1.55 \mu \mathrm{m}$. 
Parenthetically, we would like to point out that these same structures can also lead to very high normal dispersion. Figure 2(a) depicts the total dispersion of an $A l_{0.2} G a_{0.8} A s$ nanorod when its radius is $a=160 \mathrm{~nm}$. As one can see, the anomalous dispersion of this structure (also shown in Fig. 1(b) for $\lambda \leq 1.4 \mu \mathrm{m}$ ) is followed by a region of strong normal dispersion especially at $1.55 \mu \mathrm{m}$. In fact around $1.55 \mu \mathrm{m}$ the dispersion is approximately $\beta^{\prime \prime}=80 \mathrm{ps}^{2} / \mathrm{m}$. Thus the dispersion of a $2 \mathrm{~cm}$ long $A l G a A s$ nanorod will be sufficient to cancel that arising from a $1.25 \mathrm{~km}$ long anomalously dispersive fiber with group velocity dispersion $D=1 \mathrm{ps} / \mathrm{km} . \mathrm{nm}$. In addition Fig. 2(b) shows the group index $n_{g}=c / v_{g}$ and the effective index $n_{\text {eff }}$ of an $A l_{0.2} G a_{0.8} A s$ nanowire of radius $a=175 \mathrm{~nm}$ as a function of wavelength. Note that the group index in this case can be as high as $n_{g} \approx 5.52$ around $1.55 \mu \mathrm{m}$, which indicates "slow" light transport (because of waveguide dispersion) in spite of the fact that bulk index is 3.27 and $n_{\text {eff }}=1.56$.
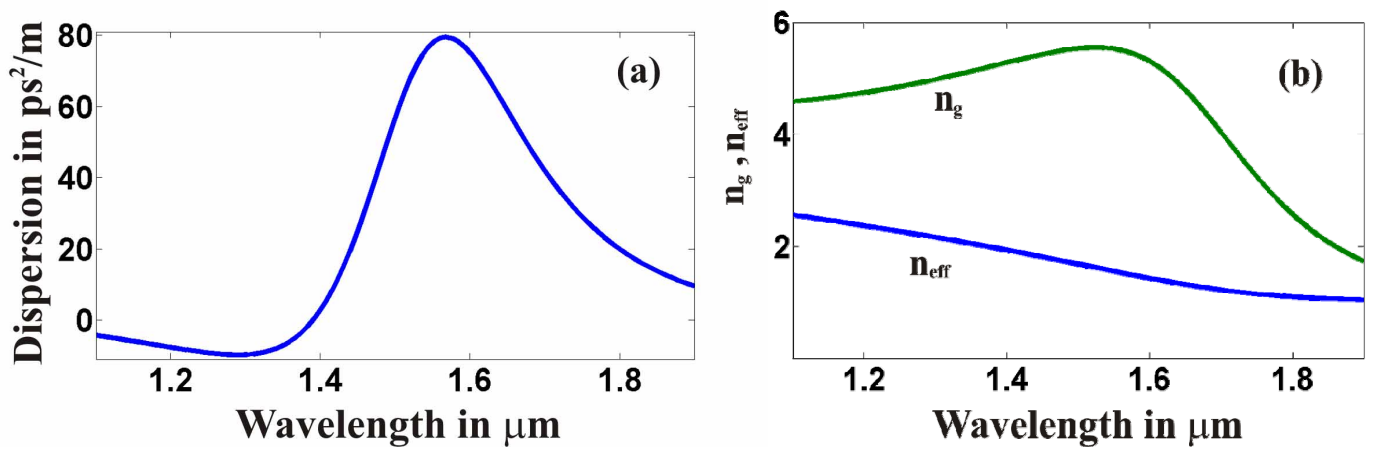

Fig. 2. (a) group velocity dispersion $\beta^{\prime \prime}$ when $a=160 \mathrm{~nm}$, (b) group and effective refractive indices of an $A l_{0.2} G a_{0.8} A s$ nanowire when $a=175 \mathrm{~nm}$.

For the subsequent discussion, we chose two operating points based on the designs (C) and (B) shown in Fig. 1(b). In particular the design (C) is used at $1.55 \mu \mathrm{m}$ where the third-order dispersion is very small whereas design (B) is used at $1.5 \mu \mathrm{m}$ where cubic dispersive effects become appreciable. The nanowire of case (C) is single-moded for $\lambda \geq 1.57 \mu \mathrm{m}$ whereas that of (B) is monomode for $\lambda \geq 1.42 \mu \mathrm{m}$.

\section{Soliton effects in AlGaAs nanowires}

Nonlinear pulse propagation in such nanowire structures is modeled using the evolution equation [22]:

$$
i \frac{\partial \varphi}{\partial z}+i \alpha \varphi+\sum_{m=2}^{\infty} \frac{i^{m} \beta^{(m)}}{m !} \frac{\partial^{m} \varphi}{\partial T^{m}}+\frac{\omega_{o} n_{2}}{2 c}\left[|\varphi|^{2} \varphi+\frac{i}{\omega_{o}} \frac{\partial\left(|\varphi|^{2} \varphi\right)}{\partial T}\right]+i \alpha_{2}|\varphi|^{2} \varphi+i \alpha_{3}|\varphi|^{4} \varphi=0
$$

In Eq.(3), $\varphi$ represents the pulse envelope, $T=t-z / v_{g}, \alpha$ is the linear loss coefficient, $\beta^{(m)}=\partial^{m} \beta / \partial \omega^{m}$ is the $m^{\text {th }}$ order dispersion coefficient evaluated at the carrier angular frequency $\omega_{0}=2 \pi c / \lambda, n_{2}=\left(\hat{n}_{2} n\right) /\left(2 \eta_{o}\right) \quad$ where $\hat{n}_{2}=1.3 \times 10^{-13} \mathrm{~cm}^{2} / W$ is the 
nonlinear Kerr coefficient and $n$ is the linear refractive index of $A l G a A s\left(\eta_{o}\right.$ is the free space wave impedance). $\alpha_{2}=6.5 \times 10^{-15} \mathrm{~m} / V^{2}$ and $\alpha_{3}=5.63 \times 10^{-31} \mathrm{~m}^{3} / V^{4}$ are the twophoton and three-photon absorption coefficients respectively at $\lambda \approx 1.55 \mu \mathrm{m}$ [19]. In all cases, the dispersion curve is incorporated in Eq.(3) using a dispersion Taylor series over a broad spectral range (greatly exceeding the pulse spectrum). Here the linear loss for the field is taken to be $\alpha=.25 \mathrm{~cm}^{-1}$.

Figure 3(a) shows the intensity evolution of a $200 f_{S}$ (FWHM) hyperbolic secant optical pulse when is launched into a $5 \mathrm{~mm}$ long $\mathrm{Al}_{0.2} G a_{0.8} A s$ nanowire of core radius $a=193 \mathrm{~nm}$ (corresponding to curve (C) of Fig.1(b)) at $1.55 \mu \mathrm{m}$. For this design, the quadratic dispersion $\left(\beta^{\prime \prime}=-12 p s^{2} / \mathrm{m}\right)$ dominates the propagation process (the higher-order dispersion terms are negligible) and the dispersion length is $\approx 1.5 \mathrm{~mm}$, i.e., is very small. The pulse peak power in this case is approximately $5.5 \mathrm{~W}$ (with a $75 \%$ confinement factor), corresponding to the fundamental soliton in this nanowire structure. As Fig. 3(a) clearly indicates, this soliton can be sustained over approximately 3-4 dispersion lengths, i.e. up to a distance that is ultimately determined by the linear loss of the system. Fig. 3(b) on the other hand depicts soliton compression under similar conditions when the input power of the pulse is $8 \mathrm{~W}$. For this power level, at 2 dispersion lengths, the FWHM pulsewidth becomes $175 f s$. Figure 3(c) and (d) demonstrate similar results when an $A l G a A s$ nanowire corresponding to curve (B) of Fig.1(b), is used at $1.5 \mu \mathrm{m}$. Even in this case, in spite of appreciable third-order dispersion effects and multi-photon absorption, a $200 f_{s}$ soliton forms at $2 \mathrm{~W}$, as shown in Fig. 3(c). Soliton compression is also shown for this case in Fig. 3(d) when the input power is $2.8 \mathrm{~W}$. It is important to note that in all cases ultrashort solitons in these nanowires can form within millimeters and at very low power levels.
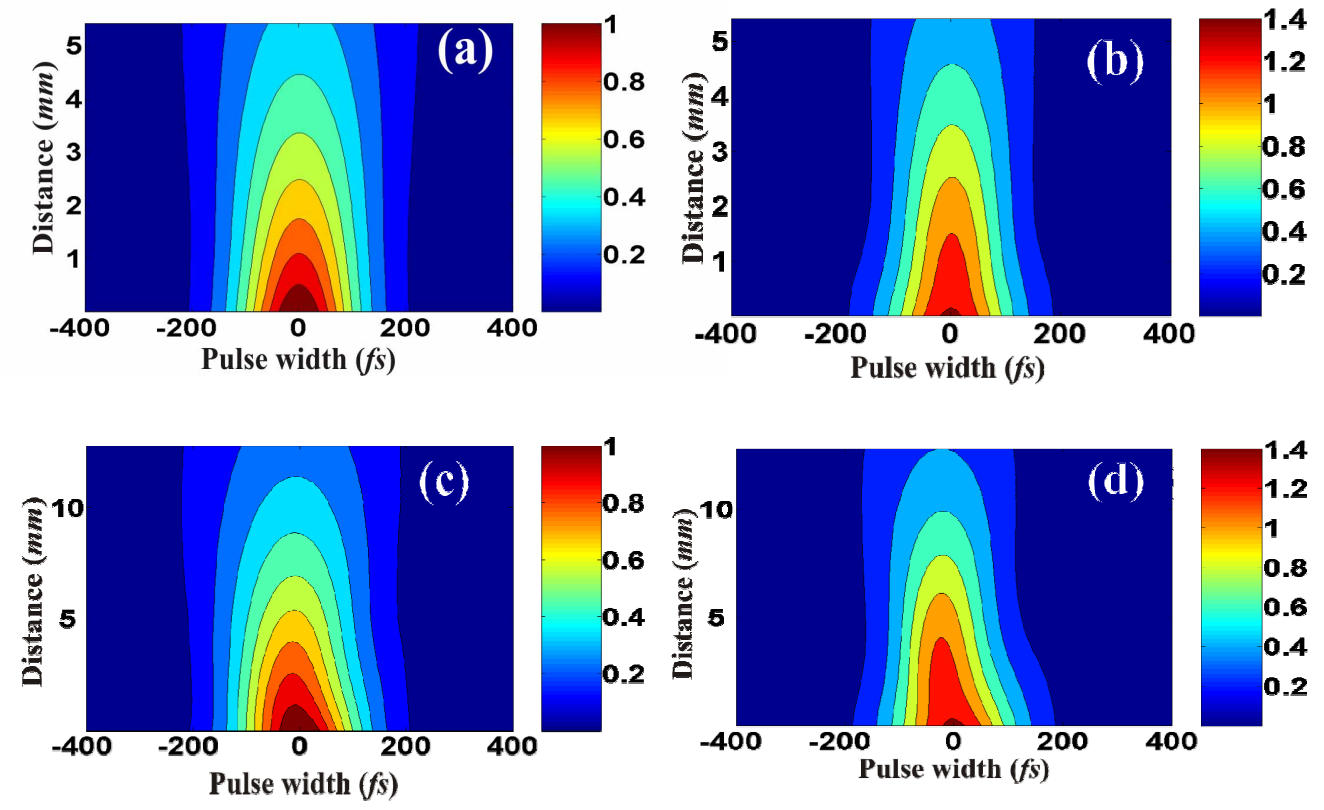

Fig. 3 Intensity evolution of a $200 \mathrm{fs}$ soliton propagating in a $193 \mathrm{~nm}$ (radius) AlGaAs nanowire when the peak power is (a) 5.5 and (b) $8 \mathrm{~W}$. Similarly, (c) and (d) depict soliton propagation and compression in a nanowire of radius $175 \mathrm{~nm}$ when the peak power is 2 and 2.8 $W$ respectively. 
Figure 4 depicts the power spectra that may result in an $A l_{0.2} G a_{0.8} A s$ nanowaveguide of radius $a=193 \mathrm{~nm}$ when is excited at $1.55 \mu \mathrm{m}$ with a $200 \mathrm{fs}$ FWHM optical pulse. When the input peak power is enough to establish an optical soliton $(5.5 \mathrm{~W})$ the power spectrum remains essentially invariant during propagation as shown in Fig. 4(a). At a higher power $(8 \mathrm{~W})$ the pulse undergoes compressions and thus its spectrum broadens (Fig. 4(b)). Figures 4(c) and (d) on the other hand depict the expected spectra and intensity profile of a higher order soliton at $L \approx 5 \mathrm{~mm}$. In this latter case, the peak power is $50 \mathrm{~W}$ and thus corresponds to an $N=3$ higher order soliton solution. The splitting behavior observed in Fig. 4(d) is attributed to multi-photon absorption.
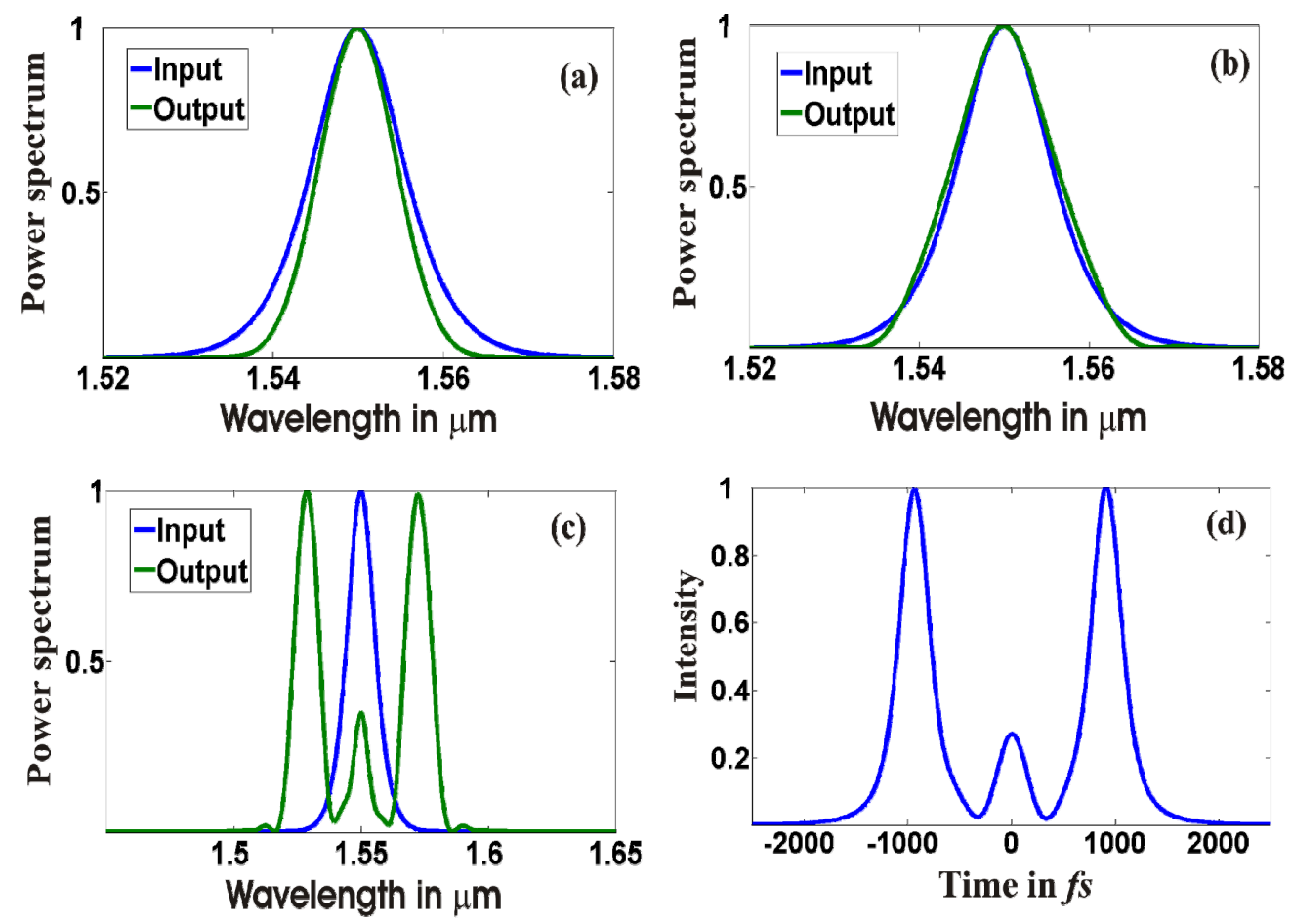

Fig. 4. (a) Input and output soliton power spectra corresponding to the case shown in (a) Fig.3 (a) ; (b) Fig.3.(b) ; $\quad$ (c) and (d) spectral generation and intensity profile for an $N=3$ soliton (50 W peak power) at $L \approx 5 \mathrm{~mm}$

In conclusion we have shown that optical solitons can exist in dispersion-inverted highlynonlinear $A l G a A s$ nanowires. These soliton waves are possible at very low power levels in spite of strong dispersion and can form in millimeter long nanowire structures. The intensity and spectral evolution of solitons propagating in such AlGaAs nanowaveguides was investigated in the presence of loss, multiphoton absorption and higher-order dispersion. 